\title{
The effect of score types on total score in trampoline gymnastics: Example of the European Championship in Sochi 2021
}

\author{
Sercin Kosova ${ }^{\mathrm{ABCDE}}$, Merve Koca Kosova ${ }^{\mathrm{ABDE}}$ \\ Department of Physical Education and Sports Teaching, Necat Hepkon Faculty of Sports Sciences, Dokuz Eylül Uni- \\ versity, Izmir, Turkey
}

Authors' Contribution: A - Study design; B - Data collection; C - Statistical analysis; D - Manuscript Preparation; E - Funds Collection

\begin{abstract}
Background

and Study Aim

Trampoline gymnastics has a versatile scoring system that has evolved and become more complex over the years. This study aimed to find the effect of the difficulty (D), execution (E), time of flight (TOF), and horizontal displacement (HD) scores on the total score in male and female trampoline gymnasts.

Material and The results of the first round in the qualification round in the individual senior category of the 27th Methods European Championships in Trampoline Gymnastics were used for evaluation. Multiple regression analysis (the enter method) was performed to build a model between the total score and D, E, TOF, and HD scores.

Results According to multiple regression analyses, respectively $E, T O F, D$, and HD scores were predictors of the total score in the men's first routine, and D, E, TOF, and HD in the men's second routine $(p<0.05)$. Although women have the same ranking as the men of the second routines, respectively $E, D, T O F$, and HD scores were predictors of the first routine $(p<0.05)$.

Conclusions: The results show that the $\mathrm{E}$ score is critical for the first routines and the $\mathrm{D}$ score is critical for the second routines. The fact that the first and second routines were constructed with different strategies as per the competition rules may have caused this result. By reminding coaches and athletes of the fact that the types of scores can affect each other, it can be suggested that they adjust their target scores according to the type of routine and the stage and type of competition.

Keywords: trampoline, difficulty, execution, time of flight, horizontal displacement
\end{abstract}

\section{Introduction}

The first appearance of the trampoline was in 1934 with the initiative of George Nissen, an American gymnast. It drew attention in a short time and was used for pilot air sense training in World War II. After development processes over the years, the trampoline became a Federation Internationale De Gymnastique (FIG) discipline in 1999 [1]. Gymnasts perform turns in different axes [2] high above the trampoline bed in combination with a high jump. Trampoline gymnastics, which was included in the Olympic program in Sydney 2000 [3], continues its development and is performed by more countries at a high level.

In trampoline gymnastics, routines with 10 different elements should be practiced without any interruptions. Many technical details such as not being able to land on the trampoline with both legs at the same time or touching any area other than the trampoline bed are considered interruptions by the judges. According to the results of the qualifying round routines, eight gymnasts qualify for the finals. In the senior category, the difficulty value of the four elements in the first routines in the qualifying round is added to the total score of the routine. The difficulty value of all 10 elements in the second routines in the qualifying round is included in the total score and gymnasts should not repeat the same elements in the second routine that have the difficulty value in the first routine [4]. Therefore, the gymnasts create their first and second routines with

\footnotetext{
(c) Sercin Kosova, Merve Koca Kosova, 2021

doi:10.15561/26649837.2021.0602
}

different strategies by considering these rules.

In addition to the difficulty (D) score, there are execution (E), time of flight (TOF), and horizontal displacement (HD) scores in trampoline gymnastics evaluations. Penalty points may be deducted by the chair of judges panel. The E score is very versatile and can be subjective [5]. It is based entirely on errors that can be perceived by the human eye, and therefore it is the type of score to which the highest number of judges contribute. The basic logic of the E score is to perform movements with minimum errors in the most perfect way. Examples of such errors are showing poor body position, bent knees, and not opening in the landing phase of the element. Judges make deductions based on the rules, and the E score is calculated using two median marks.

The TOF score was included in the evaluation to increase the objective scoring [6] because it is determined by a standard electronic device. The TOF calculation starts with the gymnast's first element. As the gymnasts jump higher, the time they stay in the air will increase, thus increasing their TOF score. The HD score is a type of score that encourages the gymnast to step on the midpoint of the trampoline during the routine. Points are deducted or not for each element landing, depending on where the feet touch from the lines, which determine the distance in the trampoline bed [7]. This score type was the last added criterion for calculating the total score in trampoline gymnastics [4].

The first European Championship in trampoline 
gymnastics was held in Paris in 1969. It continues to be organized every two years ("European Gymnastics"). The last European Championship was held between April $29^{\text {th }}$ and May $2^{\text {nd }}$, 2021, in Sochi, Russia. Considering that large events could not be organized for about a year due to the effects of COVID-19, this championship was an important meeting for many federations. The fact that the Olympics will be held approximately 3 months after this championship is also important in terms of evaluating the competitors. As explained above, scoring in trampoline gymnastics is performed with more than one score type. It is important to know which of these score types is more effective in different types of routines grades to guide athletes and trainers. In this study, the aforementioned evaluation was made by using the results of the European Championship, which is a high-level competition and has high participation. The aim of this study was to investigate the effect of the types of scores (D, E, TOF, HD) on the total score in male and female trampoline gymnasts.

\section{Materials and Methods}

Participants.

The numbers of senior male and female gymnasts participating in the individual competition were 46 and 32 , respectively. Routines in which 10 elements could not be completed are excluded from the evaluation. Accordingly, in the men's competition, 41 routines were evaluated in the first routine and 33 routines were evaluated in the second routine. For women, this number was 28 for the first routine and 26 for the second routine.

Procedure.

The data obtained in the $27^{\text {th }}$ European Championships in Trampoline Gymnastics (April 29 ${ }^{\text {th }}$ - May 2 ${ }^{\text {nd }}, 2021$, Sochi, Russia) were used in the study. The first-round results of the qualification round of individual competition of the male and female gymnasts competing in the senior category were evaluated. The first round of the qualifying round consisted of two routines. The final score is calculated by collecting four score types (E, D, TOF, HD) in all routines. However, in line with the rules, only four D-point elements are added to the total score in the first routine, whereas the $\mathrm{D}$ points of all 10 elements in the second routines are included in the total score. The results of the competition were obtained from the website of SPORTTECH Ltd, the official collaborator of European Gymnastics [8].

Statistical Analyses.

Descriptive analyses were performed, and the results were expressed as mean and standard deviation for the variables. The normality of the variables was examined using the Shapiro-Wilk test and normal distribution was observed $(p>0.05)$. The independent sample t-test was used to compare the score types of the first and second routines according to sex. The correlation between the total score and D, E, TOF, and HD scores was evaluated using Pearson's correlation analysis. Multiple regression analysis assumptions were tested. There was a linear relationship between the independent variables and the dependent variable. Multiple linearity was appropriate [9]. VIF values were within normal limits [10]. Covariance was appropriate. 'Mahalanobis distance' [11], 'Cook's distance' [12] and 'centered leverage value' were in the correct range of values. Multiple regression analysis was performed to build a model between the total and E, D, TOF, and HD scores, the enter method was used as the variable selection method. Model fitting performance was assessed using the coefficient of determination $\left(\mathrm{R}^{2}\right)$. Regression coefficients and their $95 \%$ confidence intervals (CI) are also given. A p-value of $<0.05$ was used as the level of statistical significance. The IBM SPSS Statistics for Windows Ver. 20 software program (Armonk, NY: IBM Corp., 2011) was used for the statistical analyses.

\section{Results}

There were significant differences between male and female gymnasts in all score types except for the HD score of the first routine and E and HD score of the second routine (Table 1).

Multiple regression analysis was run to predict the men's first routine total score from E, D, TOF, and HD. These variables statistically significantly predicted first routine total scores, $\mathrm{F}(4,36)=1568.52, \mathrm{p}<0.001$, $\mathrm{R}^{2}=0.994$. Multiple regression analysis was run to predict the men's second routine total score from E, D, TOF, and HD. These variables statistically significantly predicted second routine total scores, $\mathrm{F}(4,28)=8555.44$, $\mathrm{p}<0.001$,

Table 1. Comparisons of scores of the gymnasts' first and second routines

\begin{tabular}{|c|c|c|c|c|c|c|}
\hline & 1st Routines & & & 2nd Routines & & \\
\hline & Mean $\pm S D$ & & & Mean $\pm S D$ & & \\
\hline Score Types & $\begin{array}{l}\text { Women } \\
n=28\end{array}$ & $\begin{array}{l}\text { Men } \\
n=41\end{array}$ & $\mathbf{p}$ & $\begin{array}{l}\text { Women } \\
n=26\end{array}$ & $\begin{array}{l}\text { Men } \\
n=33\end{array}$ & p \\
\hline $\mathbf{E}$ & $16.86 \pm 1.15$ & $17.39 \pm 1.00$ & $0.045^{*}$ & $14.94 \pm 1.07$ & $15.24 \pm 0.90$ & 0.238 \\
\hline D & $4.20 \pm 1.05$ & $5.58 \pm 0.55$ & $0.001^{* *}$ & $11.81 \pm 1.95$ & $15.88 \pm 1.43$ & $0.001^{* *}$ \\
\hline HD & $9.22 \pm 0.25$ & $9.33 \pm 0.28$ & 0.131 & $9.06 \pm 0.19$ & $9.12 \pm 0.33$ & 0.359 \\
\hline TOF & $15.83 \pm 0.67$ & $17.56 \pm 0.69$ & $0.001^{* *}$ & $15.05 \pm 0.44$ & $16.61 \pm 0.55$ & $0.001^{* *}$ \\
\hline TOTAL & $46.10 \pm 2.06$ & $49.86 \pm 1.85$ & $0.001^{* *}$ & $50.74 \pm 2.80$ & $56.85 \pm 2.34$ & $0.001^{* *}$ \\
\hline
\end{tabular}

${ }^{*} p<0.05, * *^{*}<0.005$, E: Execution score, D: Difficulty score, HD: Horizontal Displacement score, TOF: Time of Flight score, Total: Total score 
$\mathrm{R}^{2}=0.999$. The results are presented in Table 2 .

First Routine Total Points:

$-1.105+1.017 * \mathrm{E}+0.96 * \mathrm{D}+1.085 * \mathrm{HD}+1.014 * \mathrm{TOF}$

Second Routine Total Points:

$-0.168+0.995 * \mathrm{E}+0.992 * \mathrm{D}+1.046 * \mathrm{HD}+0.996 * \mathrm{TOF}$

Multiple regression analysis was run to predict the women's first routine total score from E, D, TOF, and HD. These variables statistically significantly predicted first routine total scores, $\mathrm{F}(4,23)=31475.50, \mathrm{p}<0.001, \mathrm{R}^{2}=$ 0.999 .

Multiple regression analysis was run to predict the women's second routine total score from E, D, TOF, and HD. These variables statistically significantly predicted second routine total scores, $\mathrm{F}(4,21)=283.23, \mathrm{p}<0.001, \mathrm{R}^{2}=$ 0.978 . The results are presented in Table 3.

First Routine Total Points:

$-0.369+0.979 * \mathrm{E}+0.995 * \mathrm{D}+0.989 * \mathrm{HD}+1.053 * \mathrm{TOF}$

Second Routine Total Point:
$-2.248+1.052 * \mathrm{E}+1.116 * \mathrm{D}+1.319 * \mathrm{HD}+0.807 * \mathrm{TOF}$

In both male and female gymnasts, the highest correlation in the first routine was between $\mathrm{E}$ and total score. In the second routine, the highest correlation was found between D and total score (Table 4).

\section{Discussion}

This study focused on defining the effects of different score types on the total score in trampoline gymnastics. There are some similar studies in the literature on artistic gymnastics, one of the other Olympic branches of gymnastics [13-16], and trampoline gymnastics [5,17].To our knowledge, no study in the literature has investigated the effect of score types and included the HD score, which is added to the evaluation in the last cycle. Therefore, the results of this study will fill an important gap in the literature. The most important finding of the study was that the E score was the highest predictor in the first

Table 2. Multiple regression analyses for total score in men gymnasts

\begin{tabular}{|c|c|c|c|c|c|c|c|}
\hline & Sta.Coe & & & $95 \% \mathrm{Cl}$ & & ANOVA & \\
\hline Score Types & $\beta$ & $\mathbf{t}$ & $\mathbf{p}$ & Min-Max & $\mathbf{R}^{\mathbf{2}}$ & $\mathbf{F}$ & $\mathbf{p}$ \\
\hline$E_{1 s t R}$ & 0.550 & 30.788 & $<.001$ & [0.950-1.083] & \multirow{6}{*}{0.994} & \multirow{6}{*}{1568.52} & \multirow{4}{*}{$<0.001$} \\
\hline$D_{1 s t R}$ & 0.289 & 19.464 & $<.001$ & [0.860-1.060] & & & \\
\hline $\mathrm{HD}_{1 \mathrm{stR}}$ & 0.168 & 10.826 & $<.001$ & [0.882-1.289] & & & \\
\hline $\mathrm{TOF}_{1 \mathrm{stR}}$ & 0.381 & 22.132 & $<.001$ & [0.921-1.107] & & & \\
\hline Constant $_{1 \text { stR }}$ & -1.105 & -1.079 & .288 & [-3.183-0.973] & & & \multirow{6}{*}{$<0.001$} \\
\hline$E_{2 n d R}$ & 0.384 & 60.869 & $<.001$ & [0.962-1.029] & & & \\
\hline$D_{2 n d R}$ & 0.607 & 91.365 & $<.001$ & [0.970-1.014] & \multirow{4}{*}{0.999} & \multirow{4}{*}{8555.44} & \\
\hline$H D_{2 n d R}$ & 0.146 & 25.854 & $<.001$ & [0.963-1.129] & & & \\
\hline TOF $_{2 n d R}$ & 0.235 & 32.045 & $<.001$ & [0.933-1.060] & & & \\
\hline Constant $_{2 n d R}$ & -0.168 & -0.340 & .736 & [-1.179-0.844] & & & \\
\hline
\end{tabular}

$\beta$ : Standard coefficient beta except constant, 1stR: First routine, 2ndR: Second routine, E: Execution score, D: Difficulty score, HD: Horizontal Displacement score, TOF: Time of Flight score, Total: Total score

Table 3. Multiple regression analyses for the total score in female gymnasts

\begin{tabular}{|c|c|c|c|c|c|c|c|}
\hline & Sta.Coe & & & $95 \% \mathrm{Cl}$ & & ANOVA & \\
\hline Score Types & $\beta$ & $t$ & $p$ & Min-Max & $\mathbf{R}^{2}$ & $\mathbf{F}$ & p \\
\hline $\mathrm{E}_{1 \mathrm{stR}}$ & 0.546 & 134.104 & $<0.001$ & [0.964-0.994] & \multirow{6}{*}{0.999} & \multirow{4}{*}{31475.50} & \multirow{4}{*}{$<0.001$} \\
\hline$D_{1 s t R}$ & 0.510 & 164.647 & $<0.001$ & [0.983-1.008] & & & \\
\hline$H D_{1 s t R}$ & 0.120 & 39.359 & $<0.001$ & [0.937-1.041] & & & \\
\hline TOF $_{1 \text { stR }}$ & 0.341 & 84.534 & $<0.001$ & [1.027-1.078] & & & \\
\hline Constant $_{1 \text { stR }}$ & -0.369 & -1.276 & 0.215 & {$[-0.968-0.230]$} & & \multirow{6}{*}{283.23} & \multirow{6}{*}{$<0.001$} \\
\hline$E_{2 n d R}$ & 0.402 & 12.286 & $<0.001$ & {$[0.874-1.231]$} & & & \\
\hline$D_{2 n d R}$ & 0.775 & 16.041 & $<0.001$ & [0.971-1.260] & \multirow{4}{*}{0.978} & & \\
\hline$H D_{2 n d R}$ & 0.088 & 2.148 & 0.044 & [0.042-2.596] & & & \\
\hline TOF $_{2 \mathrm{ndR}}$ & 0.125 & 3.264 & 0.004 & [0.293-1.320] & & & \\
\hline Constant $_{2 n d R}$ & -2.248 & -0.365 & 0.719 & {$[-15.053-10.557]$} & & & \\
\hline
\end{tabular}

$\beta$ : Standard coefficient beta except constant, 1stR: First routine, 2ndR: Second routine, E: Execution score, D: Difficulty score, HD: Horizontal Displacement score, TOF: Time of Flight score, Total: Total score 
Table 4. The correlations between total score and other score types

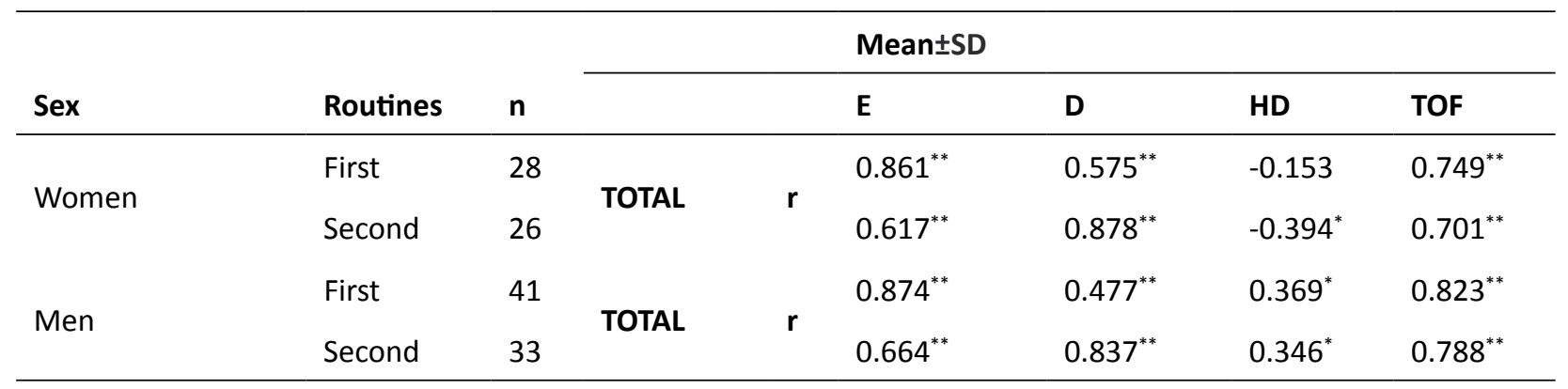

${ }^{* *} p<0.01, * p<0.05$

routines for both men and women, and the D score was the highest predictor in the second routines.

In the men's first routine, respectively TOF, D, and HD scores were effective for determining the total score after the E score. These results are not surprising. Counting the difficulty value of only four elements in the first routines caused an increase in the importance of $\mathrm{E}$ and TOF scores. In terms of homogeneity [17], D scores in the first routines may be more homogeneously distributed compared with other score types. Leskošek, Čuk and Peixoto [5] found the same importance of ranking (E, TOF, D) in men's routines at the European Championship in 2014. However, unlike the current study, the same ranking was valid in both the first and second routines. In this study, respectively D, E, TOF, and HD scores were predictors of secondroutine total scores. Gymnasts put the highest difficulty elements that they can do in their routines because the difficulty values of all 10 elements in the second routines are included in the scoring. This situation is related to the technical skills and risk-taking preferences of the athletes. It is expected that the predicting factor of the D score will increase here. It can be said that the effect of the D scores increased in this study compared with the studies in the literature based on the former evaluation formats and Code of Points $[5,17]$. This can be attributed to the increasing differences between gymnasts' D scores with technical developments and increasing contention over the years. The importance of TOF scores in men's first routines should also be emphasized. The results of the study indicate that men have higher averages in TOF scores than women. This situation allowed TOF scores to have important effect on men's first routine. Gymnasts who can jump higher will use it as a score directly and will have more time in the air to practice elements, which can help increase other score types [18].

Although the prediction order of women's second routine is the same as in the men's, there is a difference in the first routine. The D score seems more effective than the TOF score. Despite the D counting of limited elements in the first routines, it was surprising to find this result. Tactical approaches such as at which stage of the routine the D score elements will be performed, or rules such as the elements which D score is counted in the first routine, cannot be repeated in the second routine, are taken into account in the creation of the routines. Due to all these reasons and technical qualifications, the first routine D scores of female gymnasts may differ and this may lead to an increase in the decisiveness of D scores.

Especially in the first routines, the importance of the E score is apparent. Although the evaluation is quite different, it has been shown that the E score is more decisive in another branch of gymnastics [19]. Keeping the E score high requires a simple logic for the athlete to perform the movements that they can easily do, which can lead to lower D scores, and increase the jump height, thus the TOF score will also increase. While trying to keep the E score high for trainers and athletes, not being able to increase the $\mathrm{D}$ score can be one of the greatest contradictions. This contradiction is also seen in other gymnastics branches [20]. Moreover, while increasing the D score, the risk of falling or not being able to continue the routine may increase. In general, coaches and athletes can be advised to adjust their risk-taking situations according to the stage of the competition (i.e. first round of the qualifying round, second round of the qualifying round, final) while making this decision. Also, the presence or absence of opponent athletes in the competition may be reviewed when making this decision [21].

Although it is a well-known and observed situation in competitions that men have higher TOF and D scores, the E score was also found high in the first routine in this competition. In particular, female gymnasts can be recommended to develop their training programs to improve their E scores, which can be achieved by not including elements in their routines that they have not yet repeated sufficiently to perform them perfectly. The HD score remained the 4th effect by ranking for all sexes and routine types. In this score type, which is obtained by deducting mistakes out of 10 points, the fact that the gymnasts achieved close scores in this competition may have caused this result. In the literature, it has been shown that gymnasts may have lower TOF and HD scores in the second routines than in the first routines. In this context, it is important to find an optimal D score that will not negatively affect the total score [22].

\section{Conclusions}

As a result, because the routine design is made with different strategies in the qualifying round in trampoline 
gymnastics, the order of predicted total scores of the score types in the first and second routines is different. In this study, it was shown that the E score in the first routines and the $\mathrm{D}$ score in the second routines were prioritized for both men and women. All coaches and athletes can be advised to consider these findings while designing routines. Also, because all score types can be interconnected and affect each other, as discussed in the text, it seems important to determine optimal target scores for different routine types and competition types.

\section{Funding}

There is no external funding.

\section{Conflicts of interest}

There is no conflict of interest.

\section{References}

1. Federation Internationale De Gymnastique. FIG online. [Internet]. 2021. [cited 2021 May 10]. Available from https://www.gymnastics.sport/site/pages/disciplines/hist-tra. php

2. Natrup J, Bramme J, de Lussanet MH, Boström KJ, Lappe M, WagnerH.Gaze behavior of trampoline gymnasts during a back tuck somersault. Human Movement Science, 2020;70: 102589. https://doi.org/10.1016/j.humov.2020.102589

3. Vicente-Mariño M. World age group competitions (wagc) as a development pillar for trampoline gymnastics: analysing national federations' results between 1999 and 2019. Science of Gymnastics Journal. 2021; 13(1).

4. 2017-2020 Code of Points Trampoline Gymnastics. Federation Internationale De Gymnastique. FIG online. [Internet]. 2016. [cited 2021 May 10]. Available from: https://www.gymnastics.sport/site/

5. Leskošek B, Čuk I, Peixoto CJ. Inter-rater reliability and validity of scoring men's individual trampoline routines at european championships 2014. Science of Gymnastics Journal. 2018; 10(1): 69-79.

6. Ferger K, Helm F, Zentgraf K. Estimating horizontal displacement deduction in trampoline gymnastics by means of constant and variable errors of landing positions: a new gold standard? Science of Gymnastics Journal. 2020; 12(2).

7. Ferger K, Hackbarth M. New way of determining horizontal displacement in competitive trampolining. Science of Gymnastics Journal. 2017; 9(3): 303-310.

8. Sporttech. European Championships in Trampoline, Tumbling and Double Mini-Trampoline. [Internet]. 2021. [cited 2021 May 5]. Avaliable from: https://sporttech.io/ events/d8e3facd-71c4-47da-69c5-9f010f5170a8/ovs/event/ TRA

9. Berry W, Feldman S. Multiple Regression in Practice. 2455 Teller Road, Thousand Oaks California 91320 United States of America: SAGE Publications, Inc.; 1985. https://doi.org/10.4135/9781412985208

10.Allison PD. Multiple regression: A primer: Pine Forge Press. 1999.
11.De Maesschalck R, Jouan-Rimbaud D, Massart DL. The mahalanobis distance. Chemometrics and intelligent laboratory systems. 2000; 50(1): 1-18. https://doi.org/10.1016/S0169-7439(99)00047-7

12.Cook RD, Weisberg S. Residuals and influence in regression: New York: Chapman and Hall; 1982.

13.Atiković A, Kamenjašević E. The prediction of all-around event final score based on $\mathrm{d}$ and e score factors in women's artistic gymnastics. Science of Gymnastics Journal. 2021; 13(1).

14.Čuk I, Forbes W.. How apparatus difficulty scores affect all around results in men's artistic gymnastics. Science of Gymnastics Journal. 2010; 2(3): 57-63.

15.Kosova MK, Kosova S. Evaluation of scores of the 33rd and 34th Junior European Championships in Men's Artistic Gymnastics European Journal of Human Movement, 2021.

16. Massidda M, Calò CM. Performance scores and standings during the 43rd Artistic Gymnastics World Championships, 2011. Journal of Sports Sciences. 2012; 30(13): 1415-1420. https://doi.org/10.1080/02640414.2012.710759

17.Heinen T, Krepela F. Evaluating routines in trampoline gymnastics. Science of Gymnastics Journal. 2016; 8(3): 229-238.

18.Briggs K. The relationship between strength, power and trampoline jump height. Cardiff Metropolitan University; 2014.

19.Kalinski SD, Jelaska G, Atiković A. Elite female vault finals from 2008-2016. Acta Kinesiol. 2017; 11: 62-66.

20.Cuk I, Fink H, Leskosek B. Modeling the final score in artistic gymnastics by different weights of difficulty and execution. Science of Gymnastics Journal. 2012; 4(1):73.

21.Meissner L, Rai A, Rotthoff KW. The superstar effect in gymnastics. Applied Economics. 2021; 1-8. https://doi.org/10.1080/00036846.2020.1869170

22.Kosova MK, Kosova S. A comparison of time of flight and horızontal displacement scores in trampoline gymnastics routınes. Science of Gymnastics Journal, 2021;13. https://doi.org/10.52165/sgj.13.2.221-229 


\section{Information about the authors:}

Sercin Kosova; (corresponding author); https://orcid.org/0000-0003-1005-3387; sercin.kosova@gmail.com; Dokuz Eylül University; İzmir, Turkey.

Merve Koca Kosova; https://orcid.org/0000-0003-0454-2790; merve.koca@deu.edu.tr; Dokuz Eylül University; İzmir, Turkey.

Cite this article as:

Kosova S, Koca Kosova M. The effect of score types on total score in trampoline gymnastics: Example of the European

Championship in Sochi 2021. Pedagogy of Physical Culture and Sports, 2021;25(6):349-354.

https://doi.org/10.15561/26649837.2021.0602

This is an Open Access article distributed under the terms of the Creative Commons Attribution License, which permits unrestricted use, distribution, and reproduction in any medium, provided the original work is properly cited (http://creativecommons.org/licenses/by/4.0/deed.en).

Received: 15.06 .2021

Accepted: 19.08.2021; Published: 30.12.2021 